\title{
OA-016 PREVALENCE AND RISK FACTORS FOR EFAVIRENZ-BASED ANTIRETROVIRAL TREATMENT-ASSOCIATED SEVERE VITAMIN D DEFICIENCY: A PROSPECTIVE COHORT STUDY
}

Hanna Nylén, ${ }^{1}$ Abiy Habtewold, ${ }^{2}$ Eyasu Makonnen, ${ }^{2}$ Getnet Yimer, ${ }^{2}$ Jürgen Burhenne, ${ }^{3}$ Ulf Diczfalusy, ${ }^{1}$ Eleni Aklillu ${ }^{1} .{ }^{1} \mathrm{Kl}$, Sweden; ${ }^{2}$ Addis Ababa University, Ethiopia; ${ }^{3}$ University of Heidelberg, Germany

\subsection{6/bmjgh-2016-000260.24}

Background Initiation of efavirenz-based combination antiretroviral therapy (cART) is associated with Vitamin D deficiency, but the risk factors for cART-induced severe vitamin D deficiency (SVDD) and the impact of anti-tuberculosis (TB) co-treatment is not explored well.

Methods Treatment-naïve HIV patients with $(n=102)$ or without $(n=89)$ tuberculosis co-infection were enrolled prospectively and received efavirenz-based cART. In TB-HIV co-infected patients, rifampicin-based TB treatment was initiated. Plasma 25-hydroxyvitamin D (25(OH)D), cholesterol and 4-beta hydroxycholesterol concentrations were measured at baseline, and weeks 4, 16 and 48 of cART. Plasma efavirenz concentrations were determined at week 4 and 16 of cART. Genotyping for CYP2B6, CYP3A5, ABCB1, SLCO1B1, and UGT2B7 were done.

Results TB-HIV patients had significantly lower plasma 25 (OH)D3 levels than HIV-only patients at baseline. TB co-infection, low Karnofsky score, high viral load and high CYP3A activity as measured by plasma 4-beta hydroxycholesterol/ cholesterol ratios were significant predictors of low 25 $(\mathrm{OH})$ D3 levels at baseline. In HIV-only patients, initiation of efavirenz-based cART increased the prevalence of SVVD from $27 \%$ at baseline to $76 \%, 79 \%$ and $43 \%$ at weeks 4,16 and 48 of cART, respectively. The median $25(\mathrm{OH}) \mathrm{D} 3$ levels declined from baseline by $-40 \%,-50 \%$ and $-14 \%$ at weeks 4,16 and 48 of cART, respectively. In TB-HIV patients, prior TB therapy had no influence on $25(\mathrm{OH}) \mathrm{D} 3$ levels, but the initiation of efavirenz-based cART increased the prevalence of SVDD from $57 \%$ at baseline to $70 \%$ and $72 \%$ at weeks 4 and 16 of cART, respectively. Whereas the median plasma $25(\mathrm{OH}) \mathrm{D} 3$ declined from baseline by $-17 \%$ and $-21 \%$ at week 4 and 16 of cART, respectively. None of the genotypes were significantly associated with SVDD.

Conclusions Low plasma cholesterol, high CYP3A activity, and high plasma efavirenz concentrations are significant predictors of early efavirenz-based cART-induced SVDD. Low plasma 25 $(\mathrm{OH}) \mathrm{D} 3$ level at baseline is associated with TB co-infection and HIV diseases progression. 